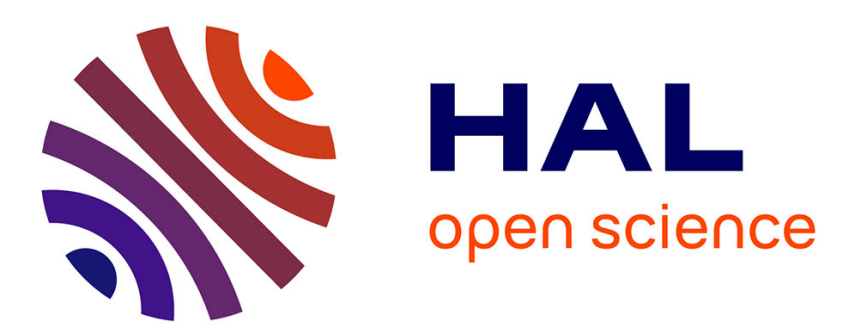

\title{
Serine hydroxymethyltransferase: a key player connecting purine, folate and methionine metabolism in Saccharomyces cerevisiae
}

Christelle Saint-Marc, Hans C Hürlimann, Bertrand Daignan-Fornier, Benoît Pinson

\section{To cite this version:}

Christelle Saint-Marc, Hans C Hürlimann, Bertrand Daignan-Fornier, Benoît Pinson. Serine hydroxymethyltransferase: a key player connecting purine, folate and methionine metabolism in Saccharomyces cerevisiae. Current Genetics, 2015, 61 (4), pp.633-640. 10.1007/s00294-015-0489-7 . hal02354935

\section{HAL Id: hal-02354935 \\ https://hal.science/hal-02354935}

Submitted on 12 Nov 2019

HAL is a multi-disciplinary open access archive for the deposit and dissemination of scientific research documents, whether they are published or not. The documents may come from teaching and research institutions in France or abroad, or from public or private research centers.
L'archive ouverte pluridisciplinaire HAL, est destinée au dépôt et à la diffusion de documents scientifiques de niveau recherche, publiés ou non, émanant des établissements d'enseignement et de recherche français ou étrangers, des laboratoires publics ou privés. 


\title{
connecting purine, folate and methionine metabolism
}

\author{
in Saccharomyces cerevisiae
}

Christelle Saint-Marc ${ }^{1,2}$, Hans C. Hürlimann ${ }^{1,2,3}$, Bertrand Daignan-Fornier ${ }^{1,2, *}$ and Benoît Pinson $^{1,2}$

${ }^{1}$ Université de Bordeaux, IBGC UMR 5095, 1 rue Camille Saint-Saëns F-33077 Bordeaux France

${ }^{2}$ Centre National de la Recherche Scientifique IBGC-CNRS UMR 5095 1, rue Camille Saint-Saëns CS 61390 F-33077 Bordeaux France

${ }^{3}$ Present address: Martin-Luther Universität, Universität Halle-Wittenberg, Institut für Biologie, Weinbergweg 10, 06120 Halle (Saale), Germany

\footnotetext{
* To whom correspondence should be addressed: Institut de Biochimie et Génétique Cellulaires, CNRS UMR 50951 rue C. Saint-Saëns CS 61390 F-33077 Bordeaux France. Tel: +33-556-999-001; E-mail: b.daignan-fornier@ibgc.cnrs.fr
}

Keywords: Nucleotides; Amino acids; One-carbon units; AICAR; Cross-pathway regulation, Serine hydroxymethyl transferase. 


\begin{abstract}
Previous genetic analyses showed phenotypic interactions between 5-Amino-4-Imidazole CArboxamide Ribonucleotide 5'-phosphate (AICAR) produced from the purine and histidine pathways with methionine biosynthesis. Here, we revisited the effect of AICAR on methionine requirement due to AICAR accumulation in the presence of the faul mutation invalidating folinic acid remobilization. We found that this methionine auxotrophy could be suppressed by overexpression of the methionine synthase Met6 or by deletion of the serine hydroxymethyl transferase gene SHM2. We propose that in a faul background, AICAR, by stimulating the transcriptional expression of SHM2, leads to a folinic acid accumulation inhibiting methionine synthesis by Met6. In addition, we uncovered a new methionine auxotrophy for the ade3 basl double mutant that can be rescued by overexpressing the SHM2 gene. We propose that methionine auxotrophy in this mutant is the result of a competition for 5,10-methylene-tetrahydrofolate (THF) between methionine and deoxythymidine monophosphate (dTMP) synthesis. Altogether, our data show intricate genetic interactions between one-carbon units, purine and methionine metabolism through fine-tuning of serine hydroxymethyl transferase by AICAR and the transcription factor Bas1.
\end{abstract}

\title{
Introduction
}

A growing number of metabolites are found to play important regulatory roles thereby directly connecting metabolic status and cellular functions. This is the case for 5-amino-4-imidazole carboxamide ribonucleotide 5'-phosphate (AICAR) which is an intermediate in the purine de novo synthesis pathway (Fig. 1) (Daignan-Fornier and Pinson 2012). In yeast, AICAR co-regulates purine synthesis and phosphate utilization by promoting interaction of the transcription factor Pho2 with either Bas1 or Pho4 (Pinson et al. 2009). In mammals, AICAR is an agonist of the AMP-activated kinase (AMPK) (Corton et al. 1995, Sullivan et al. 1994) and feeding sedentary mice with AICAriboside, an AICAR precursor, mimics muscular exercise (Narkar et al. 2008). Over-accumulation of AICAR is probably detrimental, as suggested by the multiple deficiencies associated with AICAR 
transformylase IMP-cyclohydrolase (ATIC) defect in human (Marie et al. 2004). Importantly, its et al. 2014). In yeast, AICAR accumulation is found in an ade16 ade17 double mutant lacking ATIC (Pinson et al. 2009). Massive AICAR accumulation associated to constitutive activation of the first step of the purine pathway combined to ade16 ade17 mutations abolishes yeast proliferation (Rebora et al. 2005). However, when the purine pathway is not hyper-activated, the ade16 ade17 mutant is viable but displays a yet unexplained auxotrophy for histidine (Tibbetts and Appling 2000). This auxotrophy is not dependent on the Pho2 or Bas1 transcription factors that regulate the purine and histidine pathways and can be suppressed by overexpression of the putative phosphomutase Pmu1 (Rebora et al. 2005) or by mutations resulting in decreased AICAR concentration (Hurlimann et al. 2011). Additionally, when combined with the faul mutation affecting folinic acid utilization (Fig. 1), the ade16 ade17 mutant is unable to grow in the absence of methionine (Holmes and Appling 2002). This phenotype can be suppressed by upstream mutations in the purine and histidine pathways blocking AICAR synthesis (Holmes and Appling 2002). Thus, while a triple ade16 ade17 faul mutant is auxotrophic for methionine, a quintuple ade16 ade17 faul ade2 his 4 mutant is not. Appling and coworkers proposed that the methionine auxotrophy of ade16 ade17 faul could be due to inhibition of methionine metabolism by combined elevated levels of AICAR and folinic acid (Holmes and Appling 2002). It is noteworthy that similarly to the ade16 ade17 double mutant, an ade3 mutant unable to synthesize 10-formyl-THF, a co-substrate for ATIC (Fig. 1), accumulates AICAR (Hurlimann et al. 2011) and displays methionine auxotrophy when associated with a faul knock-out mutation (Rebora et al. 2005). In this work, we used genetic approaches to explore how AICAR connects purine and methionine metabolism in yeast.

\section{Material and methods}

Yeast media

$\mathrm{SD}$ is a synthetic minimal medium containing $0.5 \%$ ammonium sulfate, $0.17 \%$ yeast nitrogen base 
without amino acids and ammonium sulfate (Difco), 2\% glucose and supplemented or not with adenine $(0.30 \mathrm{mM} ;+\mathrm{A})$, histidine $(0.06 \mathrm{mM} ;+\mathrm{H})$, leucine $(0.40 \mathrm{mM} ;+\mathrm{L})$, methionine $(0.13 \mathrm{mM} ;+$ M) and/or uracil (0.30 mM; + U). S-Adenosyl methionine, folic acid, glycine, homocysteine, or serine were added in the medium when indicated at $0.10 \mathrm{mM}, 2.20 \mathrm{mM}, 0.25 \mathrm{mM}, 0.20 \mathrm{mM}$, and $3.5 \mathrm{mM}$ respectively.

Strains and plasmids

All yeast strains are listed in Table 1 and belong to, or are derived from, a set of disrupted strains isogenic to BY4741 or BY4742 purchased from Euroscarf. Multi-mutant strains were obtained by crossing, sporulation and micromanipulation of meiosis progeny. The plasmid allowing expression of SHM2 gene under the control of a tetracycline repressible promoter (tet-SHM2; p3487) was obtained by PCR amplification of the SHM2 open reading frame using yeast genomic DNA as template and oligonucleotides 5'-CGCGGATCCACATGCCTTACACTCTAT-3' and 5'CGCCTGCAGTTACACACAGCCAATGGGTATTC-3'. The PCR product was digested with BamHI and PstI and cloned in PCM189 plasmid (Gari et al. 1997) opened with the same restriction enzymes.

Growth test

Yeast cells from an overnight pre-culture were re-suspended in sterile water at $3.10^{7}$ cells $/ \mathrm{ml}$ and submitted to $1 / 10$ serial dilutions. Drops $(5 \mu \mathrm{l})$ of each dilution were spotted on freshly prepared medium plates and were incubated at $30^{\circ} \mathrm{C}$ or $37^{\circ} \mathrm{C}$ for $48-72 \mathrm{~h}$ before imaging.

Northern blot analysis

The transcript levels of SHM2 and ACT1 were determined by northern blot analysis as described (Pinson et al. 2004). The SHM2 radiolabeled probe was obtained by PCR using oligonucleotides 5'ATGCCTTACACTCTATCCGAC-3' and 5'-CATAGCACCACGTGGACCTCTC-3' on yeast 
genomic DNA as template. The $A C T 1$ probe was already described (Denis et al. 1998).

Isolation of multicopy suppressors of the methionine auxotrophy phenotypes

Multicopy suppressors of the ade3 basl methionine auxotrophy phenotype were obtained by transforming the Y2844 strain with a plasmid multicopy library (PFL46LII backbone, $2 \mu$ LEU2; generous gift from F. Lacroute). Positive clones were obtained by replica-plating of the transformants on SD +AHU medium containing or not methionine. Thus, the multicopy plasmid (p3147) was extracted from the unique positive clone. Sequencing revealed a DNA fragment from chromosome XII (coordinates from 255850 bp to 261890 bp) containing SHM2 and REX2 genes. Similarly, multicopy suppressors of the ade3 faul methionine auxotrophy phenotype were obtained by transforming the Y2031 strain with the same plasmid multicopy library (PFL46LII backbone). Positives clones were then identified by replica-plating of the transformants on SD +AHU medium containing or not methionine. Three positive clones were so identified, all containing the MET6 encoding region. The plasmid (p3157) containing the chromosome V fragment with coordinates from 339444 bp to 342871 bp was further studied.

\section{Results}

The methionine auxotrophy due to AICAR accumulation in the faul background is dependent on Bas1 but not Pho2 or Pho4

As mentioned in the introduction, a triple ade16 ade17 faul mutant is auxotrophic for methionine due to AICAR accumulation (Holmes and Appling 2002) (Fig. 2a) and similarly, an ade3 faul double mutant is auxotrophic for methionine (Rebora et al. 2005) (Fig. 2b). Of note, as reported before, we noticed that the methionine auxotrophy was enhanced at high temperature (Rebora et al. 2005) and therefore several experiments were ran at $37^{\circ} \mathrm{C}$. As for ade 2 his 4 adel6 ade17 faul (Holmes and 
Appling 2002), we found that the ade8 his 1 upstream mutations abolishing AICAR synthesis (Fig. 1) reversed the methionine auxotrophy of an ade3 faul mutant (Fig. 2b). AICAR accumulation was thus critical for the methionine auxotrophy of these mutants. How does AICAR result in this methionine requirement phenotype? A well-known physiological effect of AICAR is to promote interaction between two pairs of transcription factors (Bas1/Pho2 and Pho4/Pho2) and thereby activate transcription of a set of about 60 genes (Pinson et al. 2009). We asked whether this documented mechanism could be responsible for the methionine auxotrophy observed in a faul mutant accumulating AICAR and thus whether the transcription factors Bas1, Pho2 and/or Pho4 were involved in the methionine requirement phenotype. This was done by combining ade3 faul with basl, pho 2 or pho4 mutations. The growth defect of the ade 3 faul double mutants in the absence of methionine was clearly not rescued by the pho2 or pho4 mutations (Fig. 2c), thus suggesting that the methionine auxotrophy is not strictly dependent on the activation of these transcription factors by AICAR. For the Bas1 transcription factor, we could not reach a conclusion in this genetic background, since we made the unexpected observation that a double ade3 bas1 mutant (Y2043) was itself auxotrophic for methionine even in the absence of the faul mutation (Fig. 2c, see section below). To circumvent the methionine auxotrophy of ade3 basl, we constructed a triple ade16 ade17 basl mutant. Growth of this AICAR-accumulating mutant was clearly not affected in the absence of methionine (Fig. 2d), indicating that for this specific phenotype, ade3 did not mimic ade16 ade17. We thus took advantage of the fact that ade16 ade17 basl did not require methionine for growth, to construct an ade16 ade17 bas 1 faul mutant that allowed us to evaluate the role of Bas1p in the ade16 ade17 faul methionine requirement. Our results establish that, by opposition to Pho2 and Pho4 (Fig. 2c), Bas1p is strictly required for methionine auxotrophy of the faul mutant under AICARaccumulating conditions (Fig. 2e). It is therefore likely that AICAR accumulation in the ade16 ade17 mutant activates a Bas1p-specific target that makes the ade16 ade17 faul triple mutant methioninerequiring. Of note, while most of the genes requiring Bas1 for their expression also require Pho2, expression of several of these genes is much more affected by the absence of Bas1 than that of Pho2 (Denis and Daignan-Fornier 1998, Denis et al. 1998). 
The methionine auxotrophy due to AICAR accumulation in the faul mutant is suppressed by over expression of MET6 and by deletion of SHM2

In order to find clues for the methionine auxotrophy of faul mutants accumulating AICAR, the ade3 faul double mutant was transformed with a genomic library carried on a multicopy plasmid to identify genes suppressing this methionine auxotrophy when overexpressed. As a result, three positive clones were isolated, all containing a plasmid allowing overexpression of the MET6 gene. One of them containing only the MET6 gene fully bypassed the methionine auxotrophy of the ade 3 faul strain (Fig. 3a). This result suggested that there is insufficient Met6p activity in faul mutants accumulating AICAR and that overexpression of MET6 can allow sufficient methionine synthesis for prototrophy. MET6 encodes methionine synthase which catalyzes the conversion of homocysteine into methionine (Fig. 1, orange box). Met6 activity requires the one-carbon unit donor, 5-methyl-THF and is thereby directly connected metabolically to Fau1 which is required for mobilization of folinic acid (5-formylTHF), a major source of one-carbon units (Fig. 1, yellow box). We reasoned that in the absence of faul, specific folate derivatives could be limiting and/or inhibitory. We first asked whether folate limitation could be responsible for methionine auxotrophy of the ade3 faul mutant. The methionine auxotrophy of ade3 faul was clearly not rescued by addition of folic acid (Fig. 3b), as an external source of folate derivatives (Fig. 1, blue box) (Holmes and Appling 2002). This result suggested that folate limitation due to folinic acid sequestration in the faul mutant is probably not the major reason for methionine auxotrophy. To address the second hypothesis, i.e. inhibition of Met6 by a one-carbon unit compound, the ade3 faul mutant was grown on various organic sulphur sources. The methionine auxotrophy of the ade3 faul mutant was clearly rescued by AdoMet, which decreases the requirement for methionine (Fig. 1, green box), while it was not rescued by homocysteine, the methionine precursor (Fig. 3c, orange box). This result indicates that methionine auxotrophy of the ade3 faul mutant is due to insufficient synthesis of methionine from homocysteine by Met6 (Fig. 1). Since the faul mutant accumulates folinic acid, and that this accumulation is further enhanced $\sim 4$ times in the ade16 ade17 faul mutant (Holmes and Appling 2002), we explored the possibility that folinic acid could have an inhibitory effect on methionine synthesis. We thus combined the ade16 ade17 faul 
mutations with a $s h m 2$ knock-out, because the serine hydroxymethyltransferase Shm2 was proposed to

be responsible for synthesis of folinic acid (Holmes and Appling 2002, Stover and Schirch 1990) which cannot be remobilized in the faul mutant (Fig. 1, yellow box). Importantly, the ade16 ade17 faul methionine auxotrophy was fully suppressed by shm2 knock-out (Fig. 3d), strongly suggesting that folinic acid synthesis via Shm2p is responsible for methionine auxotrophy, possibly through Met6p dysfunction as suggested by Holmes and Appling (Holmes and Appling 2002) and as indicated by isolation of MET6 as a multicopy suppressor gene (Fig. 3a). All together, these results suggested that the methionine auxotrophy of faul mutants could be the result of Met6 inhibition by folinic acid that is increased in cells accumulating AICAR (Holmes and Appling 2002) in which transcriptional expression of SHM2 is drastically increased (Hurlimann et al. 2011, Pinson et al. 2009).

The methionine auxotrophy of ade3 bas 1 is suppressed by overexpression of SHM2

The newly uncovered methionine ade3 bas1 auxotrophy (Fig. 2c) was further examined. As expected, it was complemented by introduction of either $A D E 3$ or BAS1 wild-type genes carried on centromeric plasmids (Fig. 4a). We then searched for multicopy suppressors that would restore methionine prototrophy. This approach allowed us to identify a unique multicopy suppressor plasmid which turned out to contain the SHM2 and REX2 genes. As SHM2 appears intimately linked to methionine auxotrophy (see previous section), we constructed a plasmid allowing expression of SHM2 alone (tetSHM2) and observed that this overexpression was sufficient to restore growth of the ade3 bas 1 mutant in the absence of methionine (Fig. 4b). This result suggests that low expression of SHM2 due to the bas1 mutation (Denis and Daignan-Fornier 1998) could lead to methionine auxotrophy when combined with ade3. It is noteworthy that the shm2 knock-out is synthetic lethal with ade3 (Rebora et al. 2005) and expression of SHM2 is activated by Bas1p at the transcriptional level (Denis and Daignan-Fornier 1998). Hence, in the ade3 bas1 double mutant, low expression of SHM2 due to the bas1 mutation (Denis and Daignan-Fornier 1998) could lead to a synthetic auxotrophy to methionine when combined to the ade3 mutation. Importantly, while the ade3 and ade16 ade 17 mutants affect a 
common step in the purine de novo pathway (Fig. 1), the ade16 ade17 bas 1 mutant is not auxotrophic bas 1 mutant is due to the combined low expression of SHM2 in the bas 1 mutant and to a specific effect of the ade3 mutation. Shm2 and Ade3 enzymes both contribute to the synthesis of 5,10methylene THF, a precursor of methyl-THF which is required for methionine synthesis (Fig. 1). The methionine auxotrophy of the ade3 bas 1 double mutant could therefore result from a limitation for 5,10-methylene THF. In addition, 5,10-Methylene THF, is utilized for dTMP synthesis and therefore methylenetetrahydrofolate reductase (Met12, Met13) and thymidilate synthase (Cdc21) compete for 5,10-methylene THF as a substrate (Fig. 1). In a double ade3 shm2 mutant, there would be no cytoplasmic route left for 5,10-methylene-THF synthesis and thus the lethality of this double mutant is probably due to the lack of dTMP synthesis which is essential for life. A partial limitation for 5,10methylene-THF in the ade3 bas 1 mutant would affect methionine synthesis but allow sufficient dTMP synthesis to maintain viability. Accordingly, methionine auxotrophy of the ade3 bas 1 mutant was rescued by AdoMet, which diminishes the requirement for methionine (Fig. 1), but not by homocysteine which requires methylene THF to be metabolized to methionine (Fig. 4c). Thus, by supplying AdoMet to the cell, the residual methionine synthesis would become sufficient to allow growth in the absence of exogenous methionine (Fig. 1). By contrast, medium supplementation with folic acid, an external precursor of folate derivatives which requires Shm2 to be metabolized to 5,10methylene-THF (Fig. 1), could not rescue the methionine auxotrophy (Fig. 4d).

Shm2 a key enzyme at the crossing point between purine, methionine and folate metabolisms

In this work, we have found that the shm2 knock-out suppressed the methionine auxotrophy of the ade16 ade17 faul mutant (Fig. 3d), while on the opposite, SHM2 overexpression suppressed the methionine auxotrophy of the ade3 bas 1 mutant (Fig. 4a). Consistently with the suppression of the methionine auxotrophy by basl but not pho2 knock-out (Fig. 2c), we found that SHM2 transcriptional expression is much more dependent on Bas1 than on Pho2 (Fig. 5a). This result establishes that the 
ade 3 faul methionine auxotrophy is the result of the transcriptional activation of SHM2 by Bas 1 in strains accumulating AICAR. Hence, both too low and too high expression of SHM2 can result in a methionine requirement in the ade3 background. Accordingly serine and glycine, which can be substrates or products of Shm2 (Fig. 1), had very different effects on the ade3 faul and ade3 bas 1 mutants (Fig. 5b). Indeed glycine rescued the methionine auxotrophy of ade3 faul. By contrast, serine rescued the methionine auxotrophy of ade3 bas1 (Fig. 5b). These results suggest that the serine/glycine balance favoring either the forward or the reverse reaction catalyzed by Shm 2 can result in very different phenotypical outcomes. In conclusion, all these results point to the central role of the serine hydroxymethyl transferase Shm2 in the crosstalk between purine, methionine and folate pathways.

\section{Discussion}

In this paper we show that the methionine auxotrophy of ade3 basl and ade 3 faul double mutants is due to inadequate expression of SHM2 which is too low in the ade 3 bas 1 mutant and too high in the ade3 faul mutant. Our results point to regulation of SHM2 expression as a critical controlling step connecting purine, methionine and one-carbon metabolism in yeast and potentially in other organisms. The main role for $\mathrm{Shm} 2$ in this process appears to be folate inter-conversion required to maintain proper amount of folate intermediates for methionine synthesis.

The newly uncovered methionine auxotrophy of the ade3 bas 1 double mutant can be rescued by overexpression of Shm2. We propose that methionine auxotrophy observed for this mutant results from a competition for 5,10-methylene-THF between two branches of the pathway, methionine and dTMP synthesis (Fig. 1). On the opposite, the knock-out of SHM2 can efficiently suppress the methionine requirement of a faul mutant accumulating AICAR. Importantly both ade 3 faul and ade3 bas 1 mutants were rescued by methionine or by S-adenosylmethionine but not by homocysteine (Fig. $3 c$ and $4 c$ ) strongly suggesting that these mutants affect the methionine synthetase step either by inhibiting Met6 activity, as proposed for ade3 faul, or by limiting its supplying with 5,10-methyleneTHF co-substrate, as proposed for ade3 basl. All together our data show intricate genetic interactions 
between purine, one-carbon units and methionine metabolism through fine-tuning of serine hydroxymethyl transferase.

The Bas1 transcription factor is a critical regulator of SHM2 expression (Denis and DaignanFornier 1998, Subramanian et al. 2005) and responds to both AICAR (Pinson et al. 2009) and glycine (Subramanian et al. 2005), thus integrating these signals to properly modulate one-carbon units interconversion. Importantly, in wild-type yeast cells, physiological AICAR variations in response to adenine availability significantly impact on expression of SHM2 (3.8 fold up-regulation when adenine is lacking) (Servant et al. 2012), an effect similar to what is found for all the purine de novo pathway genes (except ADE16) (Daignan-Fornier and Fink 1992, Denis et al. 1998, Servant et al. 2012). Physiological AICAR variations thereby modulate expression of the key SHM2 gene which connects purine, methionine and folate metabolic pathways. Importantly, AICAR (also named ZMP) was recently shown to co-regulate purine and one-carbon metabolism in bacteria via a riboswitch mechanism (Kim et al. 2015). This evolutionary conservation further confirms the highly strategic role of this metabolite in cross-pathway regulation.

\section{Acknowledgments}

The authors thank Dr F. Lacroute for the gift of the genomic library. H-C. H. was supported by a fellowship from Ligue Nationale contre le Cancer.

\section{References}

Ceschin J, Saint-Marc C, Laporte J, Labriet A, Philippe C, Moenner M, Daignan-Fornier B, Pinson B. 2014. Identification of yeast and human 5-aminoimidazole-4-carboxamide-1-beta-dribofuranoside (AICAr) transporters. J Biol Chem 289: 16844-16854.

Corton JM, Gillespie JG, Hawley SA, Hardie DG. 1995. 5-aminoimidazole-4-carboxamide ribonucleoside. A specific method for activating AMP-activated protein kinase in intact cells? 
Eur J Biochem 229: 558-565.

Daignan-Fornier B, Fink GR. 1992. Coregulation of purine and histidine biosynthesis by the transcriptional activators BAS1 and BAS2. Proc Natl Acad Sci U S A 89: 6746-6750.

Daignan-Fornier B, Pinson B. 2012. 5-Aminoimidazole-4-carboxamide-1-beta-D-ribofuranosyl 5'Monophosphate (AICAR), a Highly Conserved Purine Intermediate with Multiple Effects. Metabolites 2: 292-302.

Denis V, Daignan-Fornier B. 1998. Synthesis of glutamine, glycine and 10-formyl tetrahydrofolate is coregulated with purine biosynthesis in Saccharomyces cerevisiae. Mol Gen Genet 259: 246255.

Denis V, Boucherie H, Monribot C, Daignan-Fornier B. 1998. Role of the myb-like protein bas1p in Saccharomyces cerevisiae: a proteome analysis. Mol Microbiol 30: 557-566.

Gari E, Piedrafita L, Aldea M, Herrero E. 1997. A set of vectors with a tetracycline-regulatable promoter system for modulated gene expression in Saccharomyces cerevisiae. Yeast 13: 837848.

Holmes WB, Appling DR. 2002. Cloning and characterization of methenyltetrahydrofolate synthetase from Saccharomyces cerevisiae. J Biol Chem 277: 20205-20213.

Hurlimann HC, Laloo B, Simon-Kayser B, Saint-Marc C, Coulpier F, Lemoine S, Daignan-Fornier B, Pinson B. 2011. Physiological and toxic effects of purine intermediate 5-amino-4imidazolecarboxamide ribonucleotide (AICAR) in yeast. J Biol Chem 286: 30994-31002.

Kim PB, Nelson JW, Breaker RR. 2015. An ancient riboswitch class in bacteria regulates purine biosynthesis and one-carbon metabolism. Mol Cell 57: 317-328.

Liu X, et al. 2014. Discrete mechanisms of mTOR and cell cycle regulation by AMPK agonists independent of AMPK. Proc Natl Acad Sci U S A 111: E435-444. 
Marie S, Heron B, Bitoun P, Timmerman T, Van Den Berghe G, Vincent MF. 2004. AICAribosiduria: a novel, neurologically devastating inborn error of purine biosynthesis caused by mutation of ATIC. Am J Hum Genet 74: 1276-1281.

Narkar VA, et al. 2008. AMPK and PPARdelta agonists are exercise mimetics. Cell 134: 405-415.

Pinson B, Merle M, Franconi JM, Daignan-Fornier B. 2004. Low affinity orthophosphate carriers regulate $\mathrm{PHO}$ gene expression independently of internal orthophosphate concentration in Saccharomyces cerevisiae. J Biol Chem 279: 35273-35280.

Pinson B, Vaur S, Sagot I, Coulpier F, Lemoine S, Daignan-Fornier B. 2009. Metabolic intermediates selectively stimulate transcription factor interaction and modulate phosphate and purine pathways. Genes Dev 23: 1399-1407.

Rattan R, Giri S, Singh AK, Singh I. 2005. 5-Aminoimidazole-4-carboxamide-1-beta-Dribofuranoside inhibits cancer cell proliferation in vitro and in vivo via AMP-activated protein kinase. J Biol Chem 280: 39582-39593.

Rebora K, Laloo B, Daignan-Fornier B. 2005. Revisiting purine-histidine cross-pathway regulation in Saccharomyces cerevisiae: a central role for a small molecule. Genetics 170: 61-70.

Servant G, et al. 2012. Tye7 regulates yeast Ty1 retrotransposon sense and antisense transcription in response to adenylic nucleotides stress. Nucleic Acids Res 40: 5271-5282.

Stover P, Schirch V. 1990. Serine hydroxymethyltransferase catalyzes the hydrolysis of 5,10methenyltetrahydrofolate to 5-formyltetrahydrofolate. J Biol Chem 265: 14227-14233.

Subramanian M, Qiao WB, Khanam N, Wilkins O, Der SD, Lalich JD, Bognar AL. 2005. Transcriptional regulation of the one-carbon metabolism regulon in Saccharomyces cerevisiae by Bas1p. Mol Microbiol 57: 53-69.

Sullivan JE, Carey F, Carling D, Beri RK. 1994. Characterisation of 5'-AMP-activated protein kinase 
in human liver using specific peptide substrates and the effects of 5'-AMP analogues on enzyme activity. Biochem Biophys Res Commun 200: 1551-1556.

Tibbetts AS, Appling DR. 2000. Characterization of two 5-aminoimidazole-4-carboxamide ribonucleotide transformylase/inosine monophosphate cyclohydrolase isozymes from Saccharomyces cerevisiae. J Biol Chem 275: 20920-20927. 
Table 1 Yeast strains used in this study

\begin{tabular}{|c|c|c|}
\hline Strain & Genotype & Reference \\
\hline BY4741 & MATa his $3 \Delta 1$ leu2 $\Delta 0$ met $15 \Delta 0$ ura3 $\Delta 0$ & Euroscarf \\
\hline BY4742 & $M A T \alpha$ his3 $\Delta 1$ leu2 $\Delta 0$ lys $2 \Delta 0$ ura3 $\Delta 0$ & Euroscarf \\
\hline Y1162 & 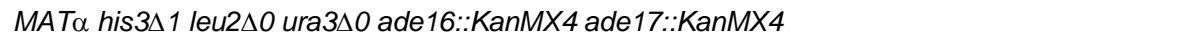 & This study \\
\hline Y2031 & MATa his $3 \Delta 1$ leu2 $\Delta 0$ ura3 $\Delta 0$ ade3::KanMX4 fau1::KanMX4 & This study \\
\hline Y2043 & MATa his $3 \Delta 1$ leu2 $\Delta 0$ ura3 $\Delta 0$ ade3::KanMX4 bas $1:: K a n M X 4$ & This study \\
\hline Y2816 & 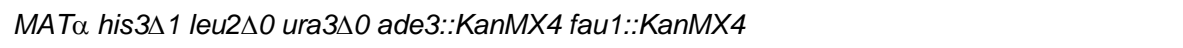 & This study \\
\hline Y2829 & 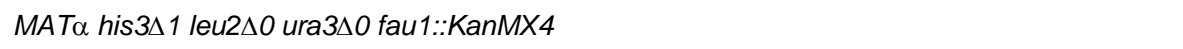 & This study \\
\hline Y2831 & MATa his $3 \Delta 1$ leu2 $\triangle 0$ ura3 $\Delta 0$ ade3::KanMX4 fau1::KanMX4 pho2::KanMX4 & This study \\
\hline Y2839 & 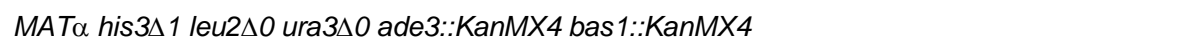 & This study \\
\hline Y2840 & 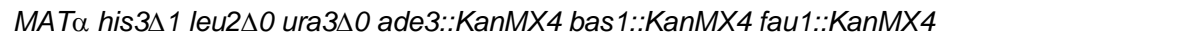 & This study \\
\hline Y2844 & MATa his3 1 leu2 $\Delta 0$ ura3 $\Delta 0$ ade3::KanMX4 bas $1:: K a n M X 4$ & This study \\
\hline Y2845 & 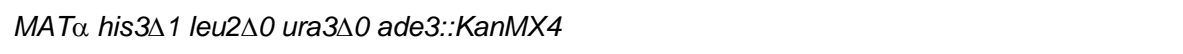 & This study \\
\hline Y2946 & $M A T \alpha$ his $3 \Delta 1$ leu2 $\Delta 0$ ura3 $\Delta 0$ & Pinson et al 2009 \\
\hline Y2954 & 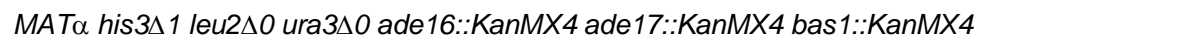 & Pinson et al 2009 \\
\hline Y4252 & 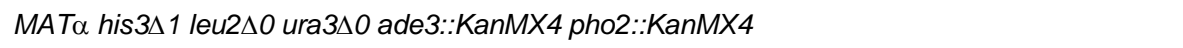 & This study \\
\hline Y4276 & 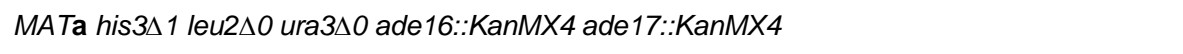 & This study \\
\hline Y4277 & 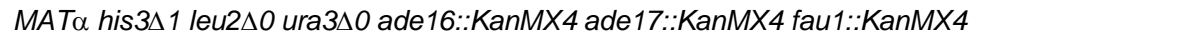 & This study \\
\hline Y4278 & 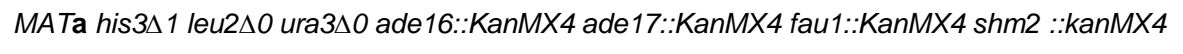 & This study \\
\hline Y4279 & 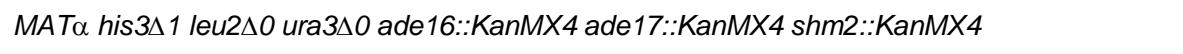 & This study \\
\hline Y4924 & 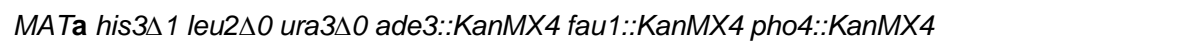 & This study \\
\hline Y4925 & 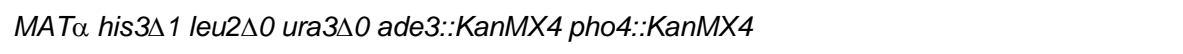 & This study \\
\hline Y4985 & MATa his $3 \Delta 1$ leu2 $\Delta 0$ ura3 $\Delta 0$ ade3::KanMX4 fau1::KanMX4 ade8::KanMX4 his1::KanMX4 & This study \\
\hline Y5613 & 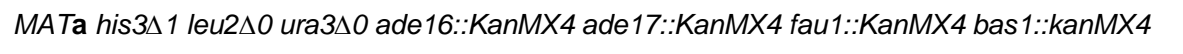 & This study \\
\hline Y7210 & 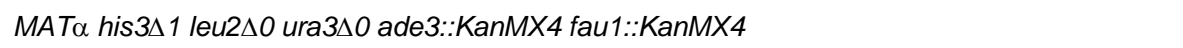 & This study \\
\hline
\end{tabular}




\section{Legends of figures}

Fig. 1 Schematic representation of the de novo purine and histidine pathways in yeast and their connection to the one-carbon unit metabolism and the methyl cycle. Only the enzymes mentioned in the text are listed in green or dark blue (Fau1). Ado: adenosine; AdoMet: S-adenosyl-methionine; AICAR: 5-amino-4-imidazole carboxamide ribonucleotide 5'-phosphate (in red); DHF: dihydrofolic acid; dTMP: deoxythymidine 5'-monophosphate; dUMP: deoxyuridine 5'-monophosphate; IMP: inosine 5'-monophosphate; PRPP: 5-phosphoribosyl-1-pyrophosphate; SAH: S-adenosyl homocysteine; SAICAR: succinyl-AICAR; S-AMP: Succinyl adenosine 5'-monophsophate. THF: tetrahydrofolic acid.

Fig. 2 AICAR accumulation leads to a Bas1-dependent methionine auxotrophy of faul mutants. a) The triple ade16 ade17 faul mutant is auxotroph for methionine. Strains: Y1162 and Y4277 b) Methionine auxotrophy of ade3 faul double mutant is abolished in the absence of AICAR. Strains: Y2816, Y2845 and Y4985 c) Deletion of $\mathrm{PHO} 2$ and $\mathrm{PHO4}$ genes does not restore growth of the ade3 fau1 mutant on methionine free medium. Strains: Y2816, Y2831, Y2043, Y2840, Y4252 and Y4925. d) The ade16 ade17 bas 1 triple mutant is not methionine auxotroph. Strains: Y1162, Y2839, Y2845, Y2946 and Y2954. e) The Bas1 transcription factor is required for methionine auxotrophy of an ade16 ade17 faul mutant. Strains: Y4276, Y5613 and Y7210. (a-e) Yeast strains were serial diluted and grown for $48 \mathrm{~h}$ at $37^{\circ} \mathrm{C}$ on SD +HLUA medium supplemented (+ Met) or not (- Met) with methionine.

Fig. 3 Overexpression of MET6 gene, S-adenosyl methionine supplementation and SHM2 gene deletion restore methionine prototrophy of faul mutants accumulating AICAR. a) Growth in the absence of methionine of the ade3 faul mutant is restored by overexpressing the methionine synthase gene MET6. Cells were serial diluted and grown on SD $+\mathrm{AHU}$ medium for $48 \mathrm{~h}$ at $37^{\circ} \mathrm{C}$ before imaging. b-c) Growth of the ade3 faul mutant on methionine-free medium is restored by AdoMet (c) but not by Folic acid supplementation (b). d) Methionine auxotrophy of the ade16 ade17 faul mutant 
is fully suppressed by deletion of the serine hydroxymethyl transferase gene SHM2. Yeast strains were

serial diluted and grown on SD +AHLU medium containing (+ Met) or not (- Met) methionine and supplemented with S-adenosyl methionine (AdoMet), Homocystein (HomoCys) or folic acid (Folic Ac.) as indicated. Plates were imaged after incubation for $48 \mathrm{~h}$ at $37^{\circ} \mathrm{C}$.

Fig. 4 The methionine auxotrophy of ade3 basl is suppressed by overexpression of the SHM2 gene or S-Adenosyl methionine supplementation. a) Complementation of the ade3 basl methionine auxotrophy by either $B A S 1$ or $A D E 3$ wild-type gene expressed from a centromeric plasmid. The Y2844 strain was transformed with a plasmid allowing expression of either BAS1 or ADE3 or by the cognate empty vector (YCp50). Transformants were spotted on a SD +AHL medium containing (+ Met) or not (- Met) methionine at $30^{\circ} \mathrm{C}$. b) Overexpression of the $S H M 2$ gene restores growth of an ade3 bas1 mutant on medium lacking methionine. The Y2043 strain was transformed with either a plasmid allowing expression of SHM2 under the control of a tetracycline repressible promoter (TetSHM2; p3487) or the cognate empty vector (pCM189). Transformants were spotted on a SD +AHL medium containing (+ Met) or not (- Met) methionine at $30^{\circ} \mathrm{C}$. c) S-adenosyl methionine supplementation is sufficient to restore growth of an ade3 bas 1 mutant (Y2043) on methionine free medium. d) Methionine auxotrophy of the ade3 basl (Y2844) mutant is not rescued by folic acid supplementation. c-d) Cells were serial diluted and spotted on SD +AHLU medium containing (+ Met) or not (- Met) methionine and supplemented with S-adenosyl methionine (AdoMet), Homocystein (HomoCys) or folic acid (Folic Ac.) when indicated. Plates were imaged after incubation for $48 \mathrm{~h}$ at $30^{\circ} \mathrm{C}$.

Fig. 5 The serine hydroxymethyl transferase Shm2 is central for connecting purine, methionine and folate metabolism. a) Transcriptional expression of the SHM2 gene monitored by northern blotting. Total RNAs were extracted from cells grown in SD +AHLU medium containing Methionine. SHM2/ACT1 ratios were determined using a phosphorimager and were set at 1 for the wild-type cells. b) Glycine and serine supplementation allows growth on methionine-free medium of ade3 faul and ade3 bas1 mutants, respectively. Yeast strains were serial diluted and grown on SD +AHLU medium 
containing (+ Met) or not (- Met) methionine and supplemented or not with glycine (+ Gly) or serine (+ Ser). Plates were imaged after incubation for $48 \mathrm{~h}$ at $30^{\circ} \mathrm{C}$. 


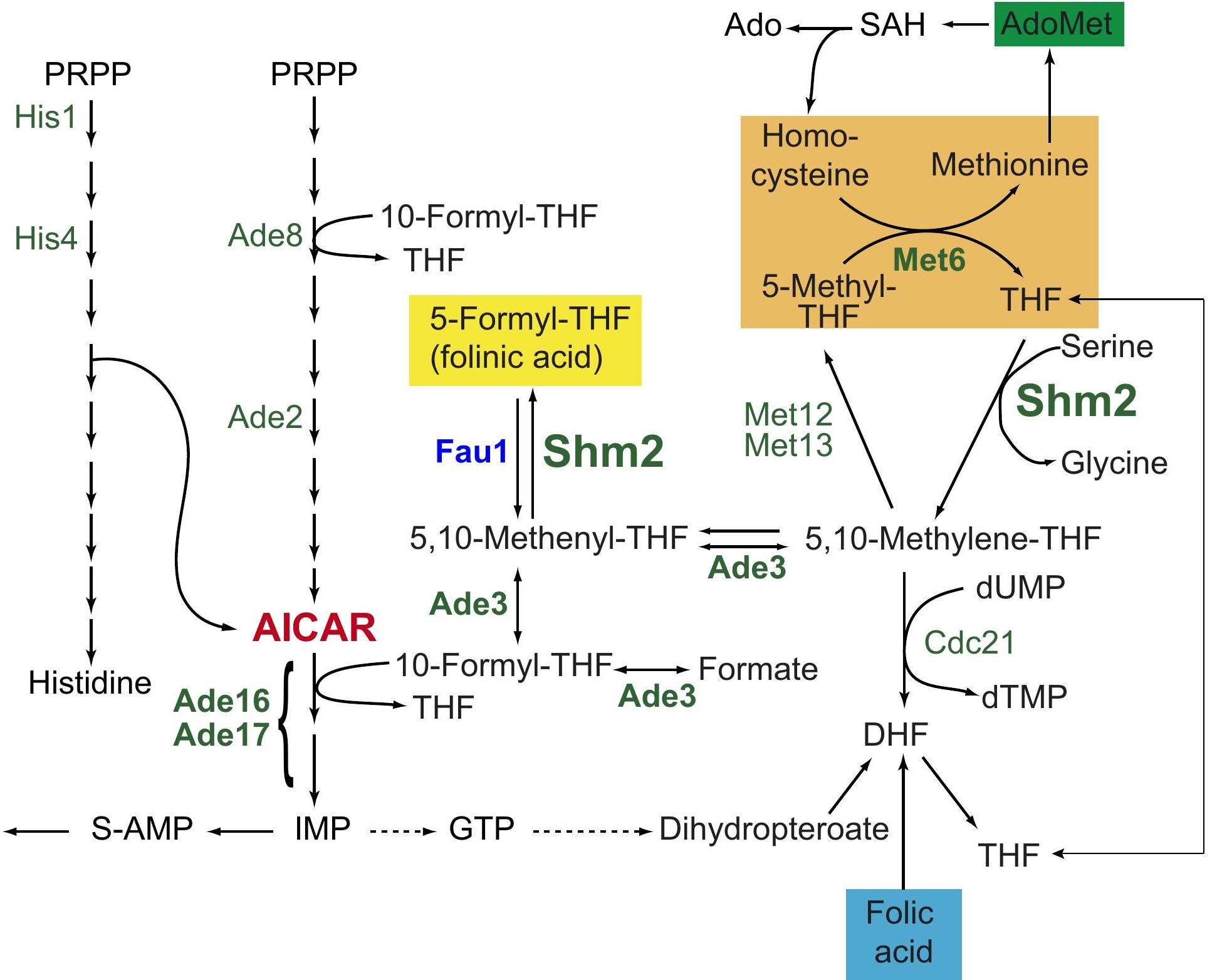

ATP

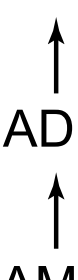

ADP

AMP

S-AMP

IMP

GTP

( 
a

\section{ade 16 ade 17} ade16 ade17 fau1

b

$$
\text { ade } 3
$$

ade8 his1 ade3 fau1

ade 3 fau 1
ade 3 fau1

C

\section{ade3 fau 1 \\ ade 3 bas 1}

ade 3 fau 1 bas 1

ade 3 pho 2

ade 3 fau1 pho2

ade3 pho 4

ade 3 fau1 pho 4

d

WT

ade16 ade17

ade3

ade16 ade17 bas 1

ade3 bas 1

e

ade16 ade 17

ade16 ade 17 fau 1 ade16 ade17 bas1 fau1
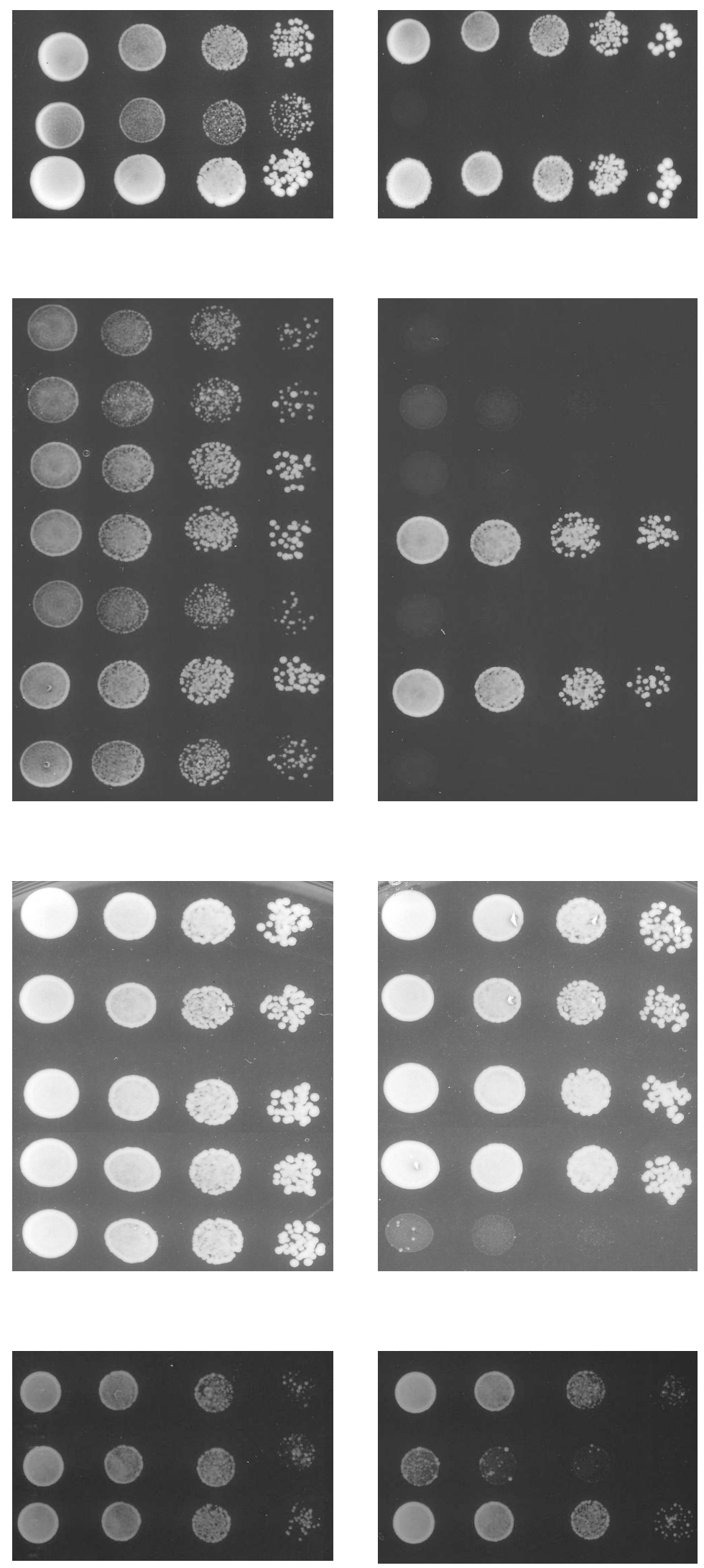
a

ade3 fau1

ade3 fau 1

\section{+ Met}

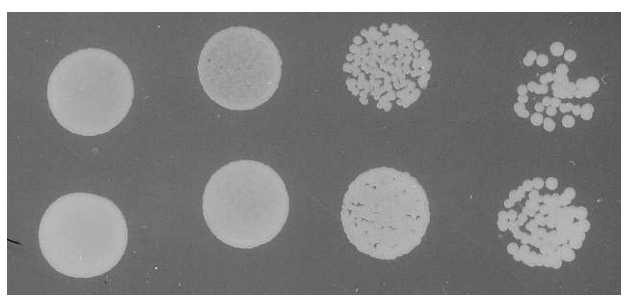

- Met

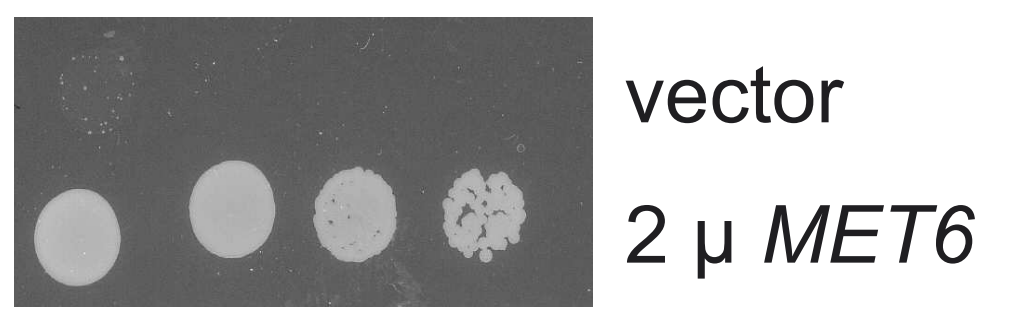

- Met

+ Met

- Met

+ Folic Ac.

ade3

fau 1

ade3 fau1
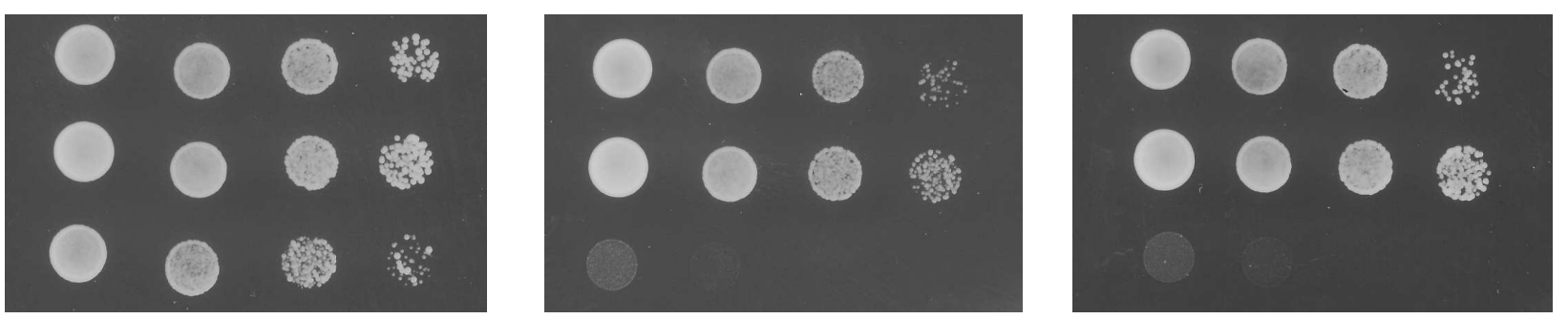

\section{Sulfur sources}

Inorganic

\section{Sulfate}

Organic Methionine

\section{WT}

ade3

fau 1

ade3 fau1

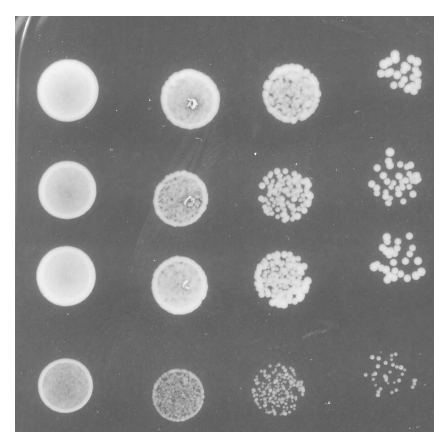

Sulfate

No

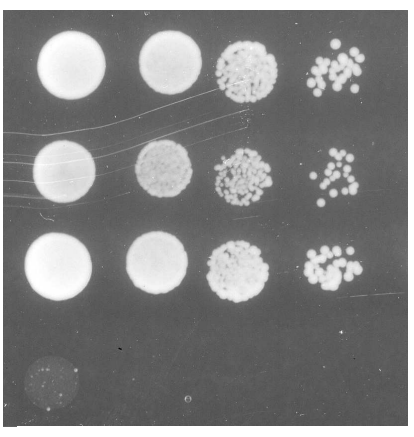

Sulfate HomoCys

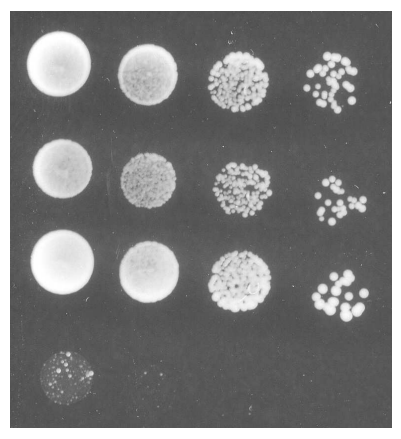

Sulfate AdoMet d

+ Met

ade16 ade17

ade16 ade17 fau1 shm2 ade 16 ade 17 fau 1 ade16 ade17 shm2
- Met 
Figure 4

a

+ Met

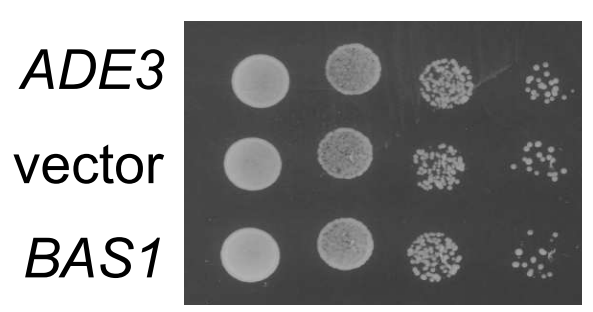

+ Met

b

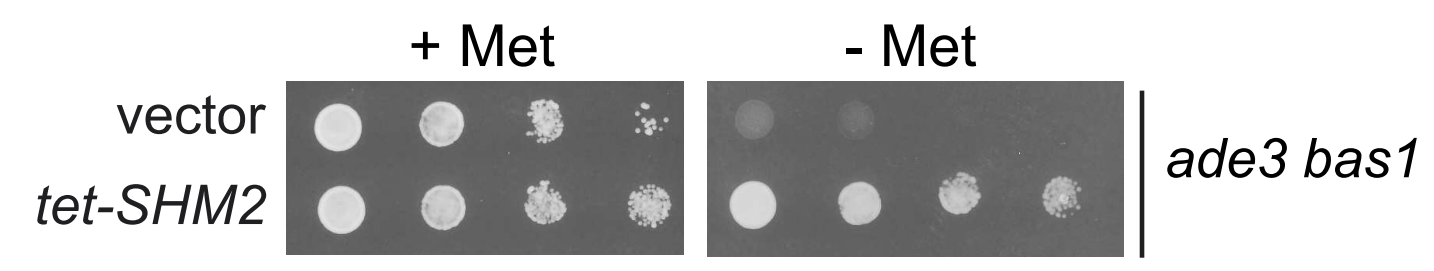

C ade3 bas 1

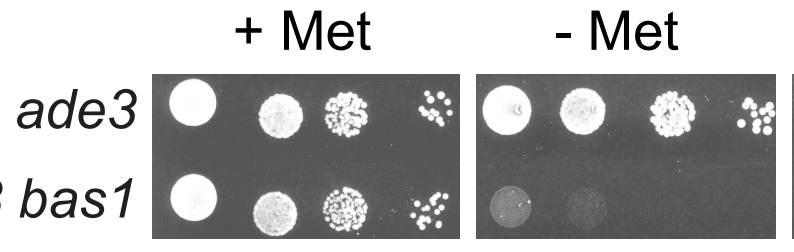

+ HomoCys + AdoMet
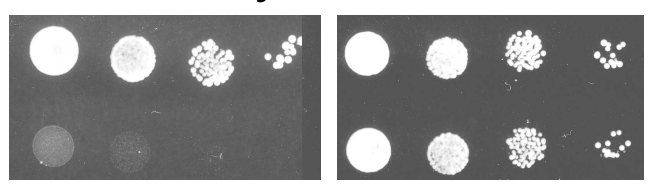

+ Met

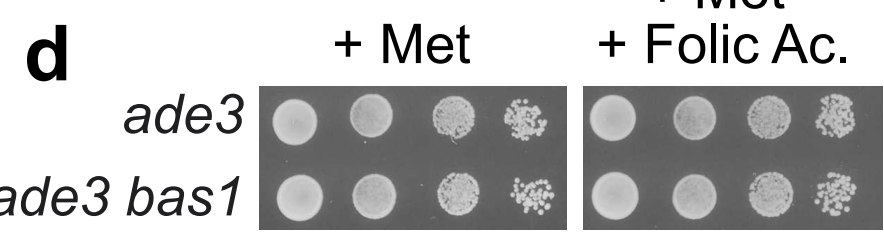

- Met + Folic Ac.
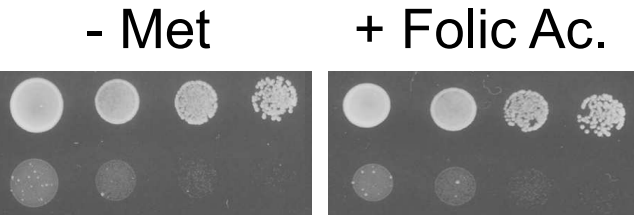
a

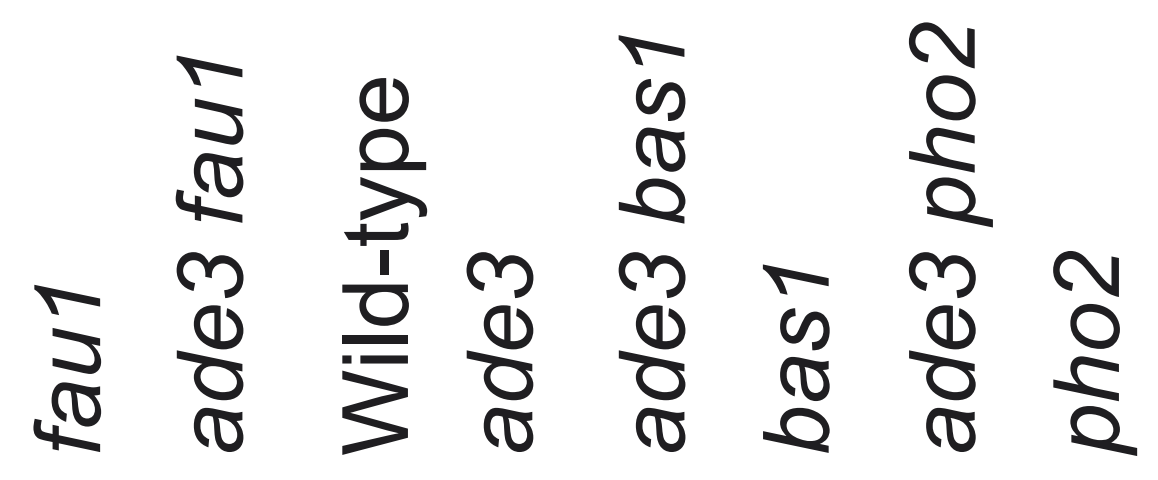

\section{SHM2}

$\begin{array}{lllllllll}S H M 2 / A C T 1 & 1.5 & 7.7 & 1.0 & 1.8 & 0.1 & 0.1 & 0.8 & 1.2\end{array}$ ACT1

b

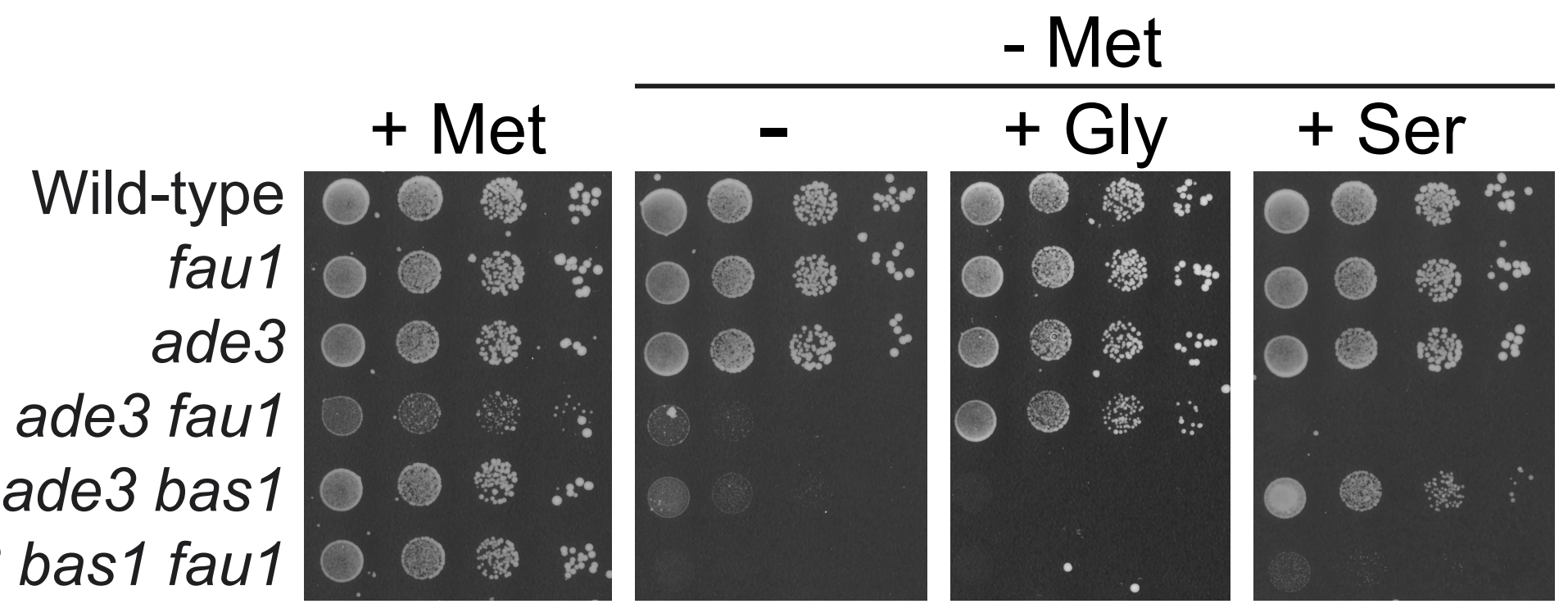
ade3 bas1 fau1 